\title{
ACTIVIDAD FÍSICA COMO ESTRATEGIA EDUCATIVA PARA MEJORAR EL RENDIMIENTO ESCOLAR
}

\author{
Alberto Ruiz Ariza \\ Departamento de Didáctica de la Expresión Musical, Plástica y Corporal. \\ Facultad de Humanidades y Ciencias de la Educación. Universidad de Jaén \\ Email: arariza@ujaen.es
}

https://doi.org/10.17060/ijodaep.2017.n1.v3.1016

Fecha de Recepción: 21 Enero 2017

Fecha de Admisión: 1 Abril 2017

\section{RESUMEN}

Actualmente existe una gran preocupación social por los bajos niveles de rendimiento escolar entre los jóvenes españoles en comparación con otros países europeos. La práctica de actividad física (AF) ha sido utilizada recientemente como estimulante para mejorar el rendimiento cognitivo de Ios jóvenes y repercutir positivamente sobre el académico (Ruiz-Ariza et al., 2017). El presente trabajo pretende conceptualizar dicha relación entre AF y cognición, así como asociar diferentes cualidades físicas con un mejor o peor rendimiento escolar, y poder así tomar decisiones sobre el tipo de AF más adecuado a fomentar desde el ámbito educativo y también familiar. Usamos regresiones lineales múltiples ajustando por edad, sexo, índice de masa corporal y nivel de estudios de las madres, para observar la relación entre cada componente de condición física, y el rendimiento escolar. Nuestros hallazgos van en la línea de la literatura previa, y muestran que una mayor condición física se asocia con mejor rendimiento escolar (Santana et al., 2016). La práctica de AF aumenta la producción de irisina en el músculo, una proteína que es clave para el cerebro y el aprendizaje. Además, un mayor fitness mejora el factor neurotrófico derivado del cerebro, el riego sanguíneo cerebral, la producción de neuronas y la conexión entre ellas (Adkins et al. 2006), mejorando las funciones ejecutivas y estimulando el aprendizaje. Como conclusión, nuestro trabajo sugiere que es necesario potenciar la práctica diaria de AF desde los Centros educativos, así como concienciar a las familias y a la sociedad en general de su promoción. Además, nuevas metodologías activas de enseñanza se presentan como una oportunidad para incluir el movimiento en cualquier asignatura y aumentar la motivación por la clase, conectar emociones, y liberar el estrés o la ansiedad, sacándole un mayor rendimiento a cada materia, y obteniendo un mayor rendimiento total.

Palabras clave

Actividad física, cognición, rendimiento escolar, metodologías activas, aprendizaje activo. 


\section{ACTIVIDAD FÍSICA COMO ESTRATEGIA EDUCATIVA PARA MEJORAR EL RENDIMIENTO ESCOLAR}

\section{ABSTRACT}

\section{Physical activity as educational strategy to improve school performance}

Currently, there is a great social concern for the low levels of school performance among the Spanish young people compared to other European countries. The practice of physical activity (PA) has recently been used as a stimulant to improve the cognitive performance in young people and to have positive repercussions on the academic performance (Ruiz-Ariza et al., 2017). The present research aims to conceptualize the relationship between PA and cognition, as well as to associate different physical qualities with better or worse school performance, and thus to be able to make decisions about the kind of PA more adequate to foment from the educative and familiar areas. We used multiple linear regressions adjusting for age, sex, body mass index, and educational level of mothers, to observe the relationship between each fitness component, and school performance. Our findings are in line with previous literature, and show that a higher physical fitness is associated with better school performance (Santana et al., 2016). The PA practice increases the production of irisine in muscle, a protein that is key to the brain and learning. In addition, improvements in fitness improves brain-derived neurotrophic factor, cerebral blood flow, neuron production and the connection between them (Adkins et al., 2006), improving executive functions and stimulating learning. In conclusion, our study suggests that it is necessary to strengthen the daily PA practice from educational centers, as well as to raise awareness among families and society about its promotion. Besides, novel active teaching methodologies are presented as an opportunity to include the movement in any subject and increase class motivation, emotions, and release stress or anxiety, making a greater performance to each subject, and obtaining a higer overall cognitive performance.

\section{Keywords}

Physical activity, cognition, school performance, active methodologies, active learning.

\section{ANTECEDENTES}

La práctica de actividad física (AF) se presenta como una gran oportunidad para mejorar la salud, prevenir enfermedades o aumentar la esperanza de vida (OMS, 2015). Además, en los últimos años, una nueva corriente neurocientífica, se está centrando en estudiar los efectos de la AF sobre el cerebro y su rendimiento, ya sea a corto 0 a largo plazo (Ruiz-Ariza et al., 2017). Estos hallazgos han llegado a los Centros educativos y cada vez son más los estudios que también relacionan la práctica de AF con mejoras en el rendimiento académico (RA) de niños y adolescentes (Mullender-Wijnsma et al., 2015).

La AF es un término muy amplio que engloba actividades de diversa índole y entre las que se encuentra la condición física (CF). El nivel de CF, sobre todo el aeróbico, se ha asociado en numerosos estudios con una mejor cognición (Aberg et al., 2009). En la última década ha habido un gran auge de estudios que estudian la relación entre CF y cognición, sin embargo los resultados aún no son concluyentes, ya que pocos estudios analizan las diferencias en función de los diferentes componentes de CF, y son escasas las propuestas de intervención centradas en el contexto real educativo en base a dichos componentes (Ardoy et al., 2014; Donnelly y Lambourne, 2011).

\section{METODOLOGÍA}

Objetivos

- Realizar una revisión de la bibliografía actual que relacione el nivel de CF con la cognición en jóvenes.

-Analizar la asociación entre CF y el RA en Matemáticas y Lengua en una muestra de adolescentes, tras ajustar por edad, IMC y status socioeducativo familiar.

- Llevar a cabo una propuesta educativa para mejorar la cognición en lo jóvenes. 


\section{Muestra de participantes}

Participaron 1164 adolescentes de 12-18 años. Los padres, directores de los Centros y profesores de EF fueron informados del propósito del mismo. Se obtuvo un consentimiento informado de los padres 0 tutores legales.

La tabla 1 presenta las características de la muestra de estudio. Un $20 \%$ de los adolescentes tenían madres con un nivel educativo universitario. Los niveles de capacidad aeróbica, fuerza en dinamometría manual y en salto horizontal, y de habilidad motora, fueron significativamente superiores en chicos que en chicas $(p<.001)$. Los chicos tenían un peso y altura superior a las chicas (todos $p<.001$ ), mientras que las chicas tenían un RA en Matemáticas y Lengua mayor que los chi$\cos$ (todos $p<.001)$.

Tabla 1.

Características de la muestra de studio

\begin{tabular}{|c|c|c|c|c|c|c|c|}
\hline & \multicolumn{2}{|c|}{$\begin{array}{c}\text { Todos } \\
(\mathrm{n}=1164)\end{array}$} & \multicolumn{2}{|c|}{$\begin{array}{l}\text { Chicos } \\
(\mathrm{n}=562)\end{array}$} & \multicolumn{2}{|c|}{$\begin{array}{l}\text { Chicas } \\
(\mathrm{n}=602)\end{array}$} & \multirow{2}{*}{ P } \\
\hline & Media & $\overline{\mathrm{DT}}$ & Media & $\overline{\mathrm{DT}}$ & Mediz & $\overline{D T}$ & \\
\hline Edad (años) & 14.49 & 1.6 & 14.54 & 1.61 & 14.46 & 1.6 & 383 \\
\hline Peso (kg) & 59.23 & 13.77 & 62.93 & 15.32 & 55.78 & 11.09 & $<001$ \\
\hline Estatura (m) & 1.63 & .08 & 1.67 & .09 & 1.60 & .06 & $<001$ \\
\hline RA Matemáticas & 6.34 & 2.05 & 6.07 & 2.17 & 6.59 & 1.97 & $<001$ \\
\hline RA Lengua & 6.32 & 1.95 & 5.94 & 2.03 & 6.68 & 1.8 & $<001$ \\
\hline Capacidad aeróbica $\left(\mathrm{VO}_{2} \max , \mathrm{mL} / \mathrm{kg} / \mathrm{min}\right)$ & 41.54 & 6.73 & 44.78 & 6.67 & 38.51 & 5.21 & $<001$ \\
\hline Fuerza media dinamometria manual, $\mathrm{kg}$ & 24.65 & 7.41 & 28.56 & 8.06 & 21.02 & 4.27 & $<001$ \\
\hline Test de salto horizontal a dos piernas, $\mathrm{cm}$ & 151.38 & 31.53 & 169.58 & 29.97 & 134.3 & 21.9 & $<001$ \\
\hline Fuerza muscular puntuación $z^{*}$ & -.06 & .84 & .46 & .84 & -.55 & .46 & $<001$ \\
\hline Habilidad motora, $\mathrm{s}$ & 12.33 & 1.3 & 11.63 & 1.1 & 12.97 & 1.12 & $<001$ \\
\hline Madres con nivel universitario (\%) & 20 & & 20.8 & & 19.3 & & .599 \\
\hline
\end{tabular}

Los valores estadísticamente significativos se identifican en negrita. RA $=$ Rendimiento Académico (escala: $0-10$ puntos). $\mathrm{VO}_{2} \max =$ Consumo máximo de oxígeno. $\mathrm{s}=$ segundos. *puntuación $z$ : se computó a través de la fuerza de dinamometría manual y la fuerza de salto horizontal a dos piernas.

\section{Instrumentos utilizados}

Se usó un método mixto mediante revisión bibliográfica, análisis cuantitativo y propuesta educativa teórico-práctica. 


\section{ACTIVIDAD FÍSICA COMO ESTRATEGIA EDUCATIVA PARA MEJORAR EL RENDIMIENTO ESCOLAR}

\section{Revisión bibliográfica}

Se usaron 2 bases de datos especializadas y se limitó la búsqueda a los últimos 10 años. Los términos de búsqueda usados se pueden observar en la tabla 2. La búsqueda se realizó en inglés.

Tabla 2.

Estrategias de búsqueda en las bases de datos.

\begin{tabular}{ll}
\hline $\begin{array}{l}\text { Bases de } \\
\text { datos }\end{array}$ & Estrategia de búsqueda \\
\hline SportDiscus & $\begin{array}{l}\text { TX ("physical fitness" OR fitness OR "physical education") and TX } \\
\text { (EBSCO) }\end{array}$ \\
& "cognitive performance" OR "academic performance" OR \\
& outcomes") and TX (adolescent* OR children OR childhood OR \\
& teenager*)
\end{tabular}

Web of ("physical education" OR "physical fitness") AND ("cognitive

Science performance" OR "academic performance" OR academic attainment

OR academic achievement OR academic outcomes)

AND (adolescent OR teenager OR children OR childhood)

\section{Investigación empírica}

Se empleó un diseño transversal. Se usó la batería ALPHA-Fitness (Ruiz et al., 2011), y para el RA se emplearon las calificaciones académicas en Matemáticas y Lengua (Ruiz-Ariza et al., 2015).

\section{Variables de confusion controladas}

El nivel socioeducativo se obtuvo a través del nivel educativo de la madre, con las siguientes opciones: sin estudios, educación primaria, educación secundaria, educación universitaria. Se preguntó directamente a las madres usando un cuestionario (Esteban-Cornejo et al., 2014). Además, se tuvo en cuenta el sexo y la edad (Ruiz-Ariza et al., 2015).

Para el peso se usó una báscula digital ASIMED tipo B clase-III (Spain) y para la altura se usó un tallímetro portátil SECA 214 (SECA Ltd., Hamburgo, Germany). IMC se calculó con la fórmula peso/talla².

\section{Análisis estadístico}

Los datos se presentan como media y DT o n (\%). Las diferencias entre sexos fueron comparadas por ANOVA de un factor para las variables continuas y con el test 2 para variables nominales. Los análisis preliminares no mostraron interacción significativa entre variables (todo $p>.05$ ); por tanto, decidimos realizar los análisis con el total de la muestra. La asociación de la CF con el RA fue analizada mediante regresión lineal. El modelo de regresión fue controlado por sexo, edad, IMC y nivel educativo de la madre. Los análisis se realizaron con el programa estadístico SPSS versión 22.0 para Windows (SPSS Inc., Chicago). El criterio para la significatividad estadística se fijó en $\mathrm{p}<$ .05 . 


\section{RESULTADOS}

El flujo de artículos en la búsqueda de resultados se puede observar en la figura 1.

Figura 1.

Flujo de artículos a través del proceso de búsqueda.

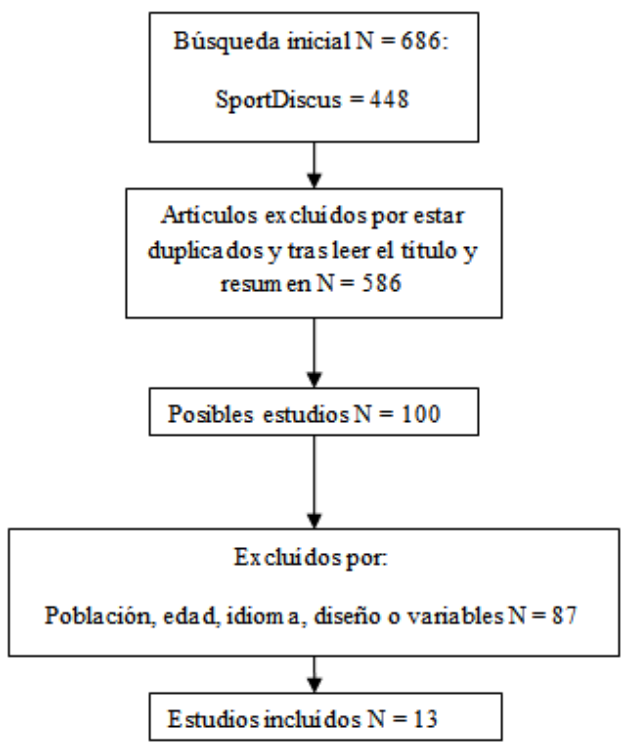

13 artículos fueron seleccionados. 7 de ellos fueron transversales (Bass et al., 2013; Coe et al., 2013; Esteban-Cornejo et al., 2014; Janak et al., 2014; Planinsec y Pisot, 2006; Sardinha et al., 2014; Welk et al., 2010), 3 de intervención (Ardoy et al., 2014; Budde et al., 2008; Travlos, 2010), y 2 longitudinales (Chen et al., 2013; London y Castrechini, 2011). 1 tuvo un doble diseño transversal y longitudinal (Aberg et al., 2009). Las variables de CF fueron la capacidad aeróbica, la fuerza muscular, la habilidad motora, la coordinación y la flexibilidad. Más del sesenta por ciento de los estudios incluyeron covariables, entre las que destacan el nivel socioeducativo de las familias, el IMC, el sexo y la edad. La mayoría de estudios mostraron una relación positiva entre CF y cognición. La capacidad aeróbica, la habilidad motora, coordinación y capacidad perceptiva son los componentes que en mayor medida se asocian con la cognición en jóvenes, sin embargo, la relación respecto a la fuerza muscular o la flexibilidad no está clara.

La tabla 3 muestra la asociación de la CF con respecto al RA tras ajustar por sexo, edad, IMC y nivel educativo de la madre. La capacidad aeróbica se asoció positivamente con Matemáticas y Lengua (todas $p \leq .001)$. La habilidad motora se asoció con Matemáticas ( $p=.008)$, y la fuerza muscular no se asoció con ninguna variable de RA ( $p>.05)$. 


\section{ACTIVIDAD FÍSICA COMO ESTRATEGIA EDUCATIVA PARA MEJORAR EL RENDIMIENTO ESCOLAR}

Tabla 3.

Asociación de los componentes de la condición física con el rendimiento académico

\begin{tabular}{lccccc}
\hline & Matemáticas & \multicolumn{2}{c}{ Lengua } \\
& $\beta$ & $\mathrm{P}$ & $\beta$ & $\mathrm{P}$ \\
\hline Capacidad aeróbica $\left(\mathrm{VO}_{2} \mathrm{max}, \mathrm{mL} / \mathrm{kg} / \mathrm{min}\right)$ & .042 & .001 & .042 & $<.001$ \\
Puntuación z de fuerza muscular* & -.033 & .746 & -.009 & .924 \\
Habilidad motora, s & -.158 & .008 & -.078 & .164 \\
\hline
\end{tabular}

Los valores estadísticamente significativos se resaltan en negrita. $\mathrm{VO}_{2} \max =$ consumo máximo de oxígeno. $\mathrm{s}=$ segundos. ${ }^{*}$ Puntuación z computada mediante fuerza manual y salto horizontal a dos piernas.

\section{DISCUSIÓN}

Los hallazgos de este trabajo están en consonancia a los de la mayoría de investigaciones previas que revelan una asociación positiva de la capacidad aeróbica con la cognición (Aberg et al., 2009; Coe et al., 2013; Sardinha et al., 2014). Algunos estudios sugieren que una capacidad aeróbica elevada podría estar asociada con mejoras en el cálculo matemático y en la resolución de problemas de aritmética (Moore et al., 2014), así como con mayor riqueza de palabras y sus significados, lo que determina un mayor nivel y control del lenguaje (Scudder et al. 2014). Además, en un estudio longitudinal en el que participaron más de un millón de adolescentes suecos, Aberg et al. (2009), demostraron que una mejora de la condición física aeróbica entre los 15 y los 18 años, predecía una mejor capacidad intelectual a los 18 años, medida con una serie de pruebas de lógica, verbales, visuoespaciales y de inteligencia técnica. La asociación entre capacidad aeróbica y cognición se puede deber a un efecto positivo de la práctica aeróbica sobre las funciones cognitivas del cerebro a nivel fisiológico, que aumentan el nivel del factor neurotrófico derivado del cerebro (BDNF). El BDNF es fundamental para proteger el sistema neuromotor e incrementar la circulación sanguínea cerebral, afectando a la mejora de la cognición (Wrann et al., 2013).

La habilidad motora también muestra asociación con la cognición. Estudios previos demuestran que está relacionada con mejor rendimiento en varias habilidades cognitivas, como el control inhibitorio, la memoria de trabajo y la atención (Ruiz-Ariza et al., 2017). En este sentido, proponer programas de intervención que incluyan el entrenamiento motor podrían mejorar la habilidad motora, y por consiguiente tener efectos positivos sobre el cerebro. Una explicación, es que la habilidad motora podrían causar sinaptogénesis, e incrementar el número de sinapsis neuronales, provocando el aumento del BDNF y la reorganización de las representaciones del movimiento en la corteza motora (Adkins et al., 2006).

\section{Propuesta educativa}

En base a nuestros hallazgos y a los estudios de Ardoy et al. (2014), Donnelly y Lambourne (2011), y Mullender-Wijnsma et al. (2015), pretendemos incluir la práctica de AF como herramienta para aumentar la cognición en el Centro educativo. Para ello, proponemos:

-En las clases de EF:

Crear unidades didácticas que tengan como eje el aumento de la capacidad aeróbica. Por ejemplo mediante la inclusión del método HIIT (Costigan et al., 2015). Además, incluir el entrenamiento 
de la habilidad motora y la coordinación mediante circuitos de agilidad y el uso de implementos como picas o raquetas.

-En las clases de Matemáticas o Lengua:

Trabajar mediante juegos dinámicos. Por ejemplo, realizar retos matemáticos en los que haya que desplazarse por la clase. Gymkhanas aeróbicas por todo el Centro en cuyas estaciones haya ejercicios de ambas asignaturas, con el objetivo de ir superando cada una de ellas hasta llegar a un trivial final con preguntas de contenidos vistos en clase.

Trabajar en Matemáticas las operaciones en función a valores reales de IMC, frecuencia cardíaca, MET, Kcal mediante pulsómetro, V02max, distancias de carrera, pasos dados al día obtenidos mediante podómetro, entre otras.

Incluir descansos de 5-8 minutos en medio de las clases para hacer algún juego de coordinación o habilidad motora con el objetivo de despertar la atención y concentración, por ejemplo: Los diez pases con la mano no dominante con una pelota de tenis, y teniendo que mantener una pierna quieta como pivote.

-Justo antes de cada clase:

Según Budde et al. (2008), 10 minutos de ejercicio coordinativo y de habilidad motora mejora la atención y la concentración, por lo que recomendamos que se dedique ese tiempo antes de cada clase a actividades de este tipo. Lo que conllevaría un aumento diario aproximado de 60 minutos de práctica de AF (cifra mínima saludable recomendada por la OMS).

-Justo antes y después de la jornada escolar:

Fomentar el desplazamiento activo para ir y volver del Centro Educativo. Estudios previos ya han demstrado los beneficios del mismo a nivel cognitivo (Martínez-Gómez et al., 2011).

-Actividad física extraescolar:

La práctica de AF aeróbica o motora en horario extraescolar también puede ser beneficiosa para el rendimiento cognitivo de los jóvenes (Esteban-Cornejo et al., 2014), por lo que el fomento de esta práctica desde los estamentos públicos y desde las familias es crucial.

\section{CONCLUSIONES}

En conclusión, la capacidad aeróbica, la habilidad motora y la coordinación pueden tener una potencial influencia sobre la cognición de los adolescentes. Sugerimos a los profesores de EF que den más importancia al componente aeróbico y motor en sus clases, y animamos a promover acciones conjuntas de forma multidisciplinar entre la asignatura de EF y las asignaturas instrumentales estudiadas, así como a fomentar el aumento de la condición física aeróbica y motora durante la adolescencia, desde el ámbito educativo, político y familiar.

\section{REFERENCIAS}

Aberg, M. A. I., Pedersen, N. L., Torén, K., Svartengren, M., Bäckstrand, B., Johnsson, T., ... Kuhn, H. G. (2009). Cardiovascular fitness is associated with cognition in young adulthood. Proceedings of the National Academy of Sciences of the United States of America, 106(49), 20906-11. http://doi.org/10.1073/pnas.0905307106

Adkins, D. L., Boychuk, J., Remple, M. S., \& Kleim, J. A. (2006). Motor training induces experiencespecific patterns of plasticity across motor cortex and spinal cord. Journal of Applied Physiology (Bethesda, Md. : 1985), 101(6), 1776-82. http://doi.org/10.1152/japplphysiol.00515.2006

Ardoy, D. N., Fernández-Rodríguez, J. M., Jiménez-Pavón, D., Castillo, R., Ruiz, J. R., \& Ortega, F. B. (2014). A physical education trial improves adolescents' cognitive performance and academic achievement: the EDUFIT study. Scandinavian Journal of Medicine \& Science in Sports, 24(1), e52-61. http://doi.org/10.1111/sms.12093 


\section{ACTIVIDAD FÍSICA COMO ESTRATEGIA EDUCATIVA PARA MEJORAR EL RENDIMIENTO ESCOLAR}

Bass, R. W., Brown, D. D., Laurson, K. R., \& Coleman, M. M. (2013). Physical fitness and academic performance in middle school students. Acta Paediatrica (Oslo, Norway: 1992), 102(8), 832-7. http://doi.org/10.1111/apa.12278

Budde, H., Voelcker-Rehage, C., Pietraßyk-Kendziorra, S., Ribeiro, P., \& Tidow, G. (2008). Acute coordinative exercise improves attentional performance in adolescents. Neuroscience Letters, 441(2), 219-223. http://doi.org/10.1016/j.neulet.2008.06.024

Costigan, S. A., Eather, N., Plotnikoff, R. C., Taaffe, D. R., \& Lubans, D. R. (2015). High-intensity interval training for improving health-related fitness in adolescents: a systematic review and meta-analysis. British Journal of Sports Medicine, 49(19), 1253-61. http://doi.org/10.1136/bjsports-2014-094490

Chen, L.-J., Fox, K. R., Ku, P.-W., \& Taun, C.-Y. (2013). Fitness change and subsequent academic performance in adolescents. The Journal of School Health, 83(9), 631-8. http://doi.org/10.1111/josh.12075

Coe, D. P., Peterson, T., Blair, C., Schutten, M. C., \& Peddie, H. (2013). Physical fitness, academic achievement, and socioeconomic status in school-aged youth. The Journal of School Health, 83(7), 500-7. http://doi.org/10.1111/josh.12058

Donnelly, J. E., \& Lambourne, K. (2011). Classroom-based physical activity, cognition, and academic achievement. Preventive Medicine, 52 Suppl 1, S36-42. http://doi.org/10.1016/i.ypmed.2011.01.021

Esteban-Cornejo, I., Tejero-González, C. M., Martinez-Gomez, D., del-Campo, J., González-Galo, A., Padilla-Moledo, C., ... Veiga, O. L. (2014). Independent and combined influence of the components of physical fitness on academic performance in youth. The Journal of Pediatrics, 165(2), 306-312.e2. http://doi.org/10.1016/i.jpeds.2014.04.044

Janak, J. C., Gabriel, K. P., Oluyomi, A. O., Peréz, A., Kohl, H. W., \& Kelder, S. H. (2014). The association between physical fitness and academic achievement in Texas state house legislative districts: an ecologic study. The Journal of School Health, 84(8), 533-42. http://doi.org/10.1111/josh.12176

London, R. A., \& Castrechini, S. (2011). A longitudinal examination of the link between youth physical fitness and academic achievement. The Journal of School Health, 81(7), 400-8. http://doi.org/10.1111/j.1746-1561.2011.00608.x

Martínez-Gómez, D., Ruiz, J. R., Gómez-Martínez, S., Chillón, P., Rey-López, J. P., Díaz, L. E., ... Marcos, A. (2011). Active commuting to school and cognitive performance in adolescents: the AVENA study. Archives of Pediatrics \& Adolescent Medicine, 165(4), 300-5. http://doi.org/10.1001/archpediatrics.2010.244

Moore, R. D., Drollette, E. S., Scudder, M. R., Bharij, A., \& Hillman, C. H. (2014). The influence of cardiorespiratory fitness on strategic, behavioral, and electrophysiological indices of arithmetic cognition in preadolescent children. Frontiers in Human Neuroscience, 8, 258. http://doi.org/10.3389/fnhum.2014.00258

Mullender-Wijnsma, M. J., Hartman, E., de Greeff, J. W., Bosker, R. J., Doolaard, S., \& Visscher, C. (2015). Improving Academic Performance of School-Age Children by Physical Activity in the Classroom: 1-Year Program Evaluation. Journal of School Health, 85(6), 365-371. http://doi.org/10.1111/josh.12259

Organización Mundial de la Salud (2015). Extraído el 02 de enero de 2016 en http://www.who.int/topics/physical_activity/es/

Planinsec, J., \& Pisot, R. (2006). Motor coordination and intelligence level in adolescents. Adolescence, 41(164), 667-76. 
Ruiz, J. R., Castro-Pinero, J., Espana-Romero, V., Artero, E. G., Ortega, F. B., Cuenca, M. M., ... Castillo, M. J. (2011). Field-based fitness assessment in young people: the ALPHA health-related fitness test battery for children and adolescents. British Journal of Sports Medicine, 45(6), 518-524. http://doi.org/10.1136/bjsm.2010.075341

Ruiz-Ariza, A., Ruiz, J., De la Torre-Cruz, M., Latorre-Román, P., \& Martínez-López, E. J. (2015). Influence of level of attraction to physical activity on academic performance of adolescents. Revista Latinoamericana de Psicologia, in press. http://doi.org/10.1016/j.rlp.2015.09.005

Ruiz-Ariza, A., Grao-Cruces, A., de Loureiro, N. E. M., \& Martínez-López, E. J. (2017). Influence of physical fitness on cognitive and academic performance in adolescents: A systematic review from 2005-2015. International Review of Sport and Exercise Psychology, 10(1), 108-133. http://doi.org/10.1080/1750984X.2016.1184699

Santana, C. C. A., Azevedo, L. B., Cattuzzo, M. T., Hill, J. O., Andrade, L. P., \& Prado, W. L. (2016). Physical fitness and academic performance in youth: A systematic review. Scandinavian Journal of Medicine \& Science in Sports. http://doi.org/10.1111/sms.12773

Sardinha, L. B., Marques, A., Martins, S., Palmeira, A., \& Minderico, C. (2014). Fitness, fatness, and academic performance in seventh-grade elementary school students. BMC Pediatrics, 14(1), 176. http://doi.org/10.1186/1471-2431-14-176

Scudder, M. R., Federmeier, K. D., Raine, L. B., Direito, A., Boyd, J. K., \& Hillman, C. H. (2014). The association between aerobic fitness and language processing in children: implications for academic achievement. Brain and Cognition, 87, 140-52. http://doi.org/10.1016/i.bandc.2014.03.016

Travlos, A. K. (2010). High intensity physical education classes and cognitive performance in eighth grade students: An applied study. International Journal of Sport and Exercise Psychology, 8(3), 302-311. http://doi.org/10.1080/1612197X.2010.9671955

Welk, G. J., Jackson, A. W., Morrow, J. R., Haskell, W. H., Meredith, M. D., \& Cooper, K. H. (2010). The association of health-related fitness with indicators of academic performance in Texas schools. Research Quarterly for Exercise and Sport, 81(3 Suppl), S16-23. http://doi.org/10.1080/02701367.2010.10599690

Wrann, C. D., White, J. P., Salogiannnis, J., Laznik-Bogoslavski, D., Wu, J., Ma, D., ... Spiegelman, B. M. (2013). Exercise induces hippocampal BDNF through a PGC-1/FNDC5 pathway. Cell Metabolism, 18(5), 649-59. http://doi.org/10.1016/j.cmet.2013.09.008 
